\title{
Efficacy of vancomycin in combination with various antimicrobial agents against clinical methicillin resistant Staphylococcus aureus strains
}

\author{
Gulseren Aktas
}

\begin{abstract}
Background: Multi-drug resistant methicillin resistant Staphylococcus aureus (MRSA) strains that have been isolated frequently worldwide have difficulties in the treatment and therefore alternative choices for the treatment of the infections are required. The aim of the study was to evaluate the interaction of various antimicrobials in combination with vancomycin against MRSA.

Methods: Twenty five clinical MRSA strains isolated in 2016 were included in the study. The interaction between vancomycin and new generation/conventional antimicrobials against MRSA strains was analyzed by E-test.

Results: All of the strains tested was found to be susceptible to vancomycin, telavancin, dalbavancin, ceptobiprole, daptomycin, linezolid, quinupristin-dalfopristin, trimethoprim-sulfamethoxazole, rifampicin and tigecycline. The susceptibility rates of the isolates were found to be high, with the lowest rate (48\%) against azithromycin. According to the fractional inhibitory concentration index results, synergistic interaction with vancomycin was determined with trimethoprim-sulfamethoxazole, azithromycin, linezolid, minocycline, dalbavancin, clindamycin in five, three, two, two, one, one and one strain(s), respectively. Additionally, all combinations studied showed additive interaction at high rates.

Conclusions: The results of the study indicate that the use of vancomycin in combination with conventional and new generation antibiotics is promising.
\end{abstract}

KEYWORDS: Conventional antibiotics, new generation antibiotics, combination, E-test, MRSA.

doi: https://doi.org/10.12669/pjms.37.1.2887

How to cite this:

Aktas G. Efficacy of vancomycin in combination with various antimicrobial agents against clinical methicillin resistant Staphylococcus aureus strains. Pak J Med Sci. 2021;37(1):151-156. doi: https://doi.org/10.12669/pjms.37.1.2887

This is an Open Access article distributed under the terms of the Creative Commons Attribution License (http://creativecommons.org/licenses/by/3.0), which permits unrestricted use, distribution, and reproduction in any medium, provided the original work is properly cited.

1. Dr. Gulseren Aktas, Ph.D. Associate Professor,

Department of Medical Microbiology,

Istanbul University,

Istanbul Faculty of Medicine,

34093, Capa-Istanbul,

Turkey.

Corresponence:

Dr. Gulseren Aktas Ph.D.

Department of Medical Microbiology,

Istanbul University,

Istanbul Faculty of Medicine,

34093, Capa-Istanbul,

Turkey.

E-mail: gulserena2001@yahoo.co.uk

* Received for Publication:

* Revision Received:

* Revision Accepted:
May 11,2020

September 18, 2020

October 15, 2020

\section{INTRODUCTION}

Multiple antibiotic resistant Gram-positive pathogens have been causing severe problems in the treatment since 1980s. ${ }^{1,2}$ Methicillinresistant Staphylococcus aureus (MRSA) remains an important cause of serious infections, particularly among hospitalized patients, and it has recently been observed in an increasing trend in community settings. ${ }^{3,4}$ Vancomycin is often recommended as the first-choice antibiotic in the treatment of MRSA infections. However, emergence of resistance during therapy has been recognized. ${ }^{5,6}$ Although vancomycin remains the preferred agent for the treatment of MRSA infections, newer antimicrobials seem to be attractive for therapeutic options. ${ }^{78} \mathrm{On}$ 
the other hand, although the newer antibiotics such as dalbavancin, telavancin, daptomycin, linezolid, tigecyclin and quinupristin/dalfopristin have been successfully used in the treatment of the infections, the presence of resistance to these antibiotics was also reported. ${ }^{9}$ The use of the combinations of antibiotics is known to increase the success of treatment with the synergistic interaction. Moreover, combination of antibiotics in the treatment has the advantage of reducing or delaying the emergence of resistant strains and reducing the toxicity. ${ }^{10}$ The aim of the present study was to evaluate the synergistic potential of vancomycin in combination with both conventional and new generation antimicrobials against MRSA strains that would give an idea for the treatment of MRSA infections.

\section{METHODS}

Twenty five MRSA strains which were randomly selected were included in the study. Each strain was isolated from different patients who were admitted to the different wards of the Istanbul University, Istanbul Faculty of Medicine's Hospital, through 2016. A total of 25 randomlyselected clinical MRSA strains were included in the study. Strains were isolated from generally blood, pass, sputum and lesser urine samples of the patients. The identification of the strains and the determination of methicillin resistance were performed using conventional methods. ${ }^{11,12}$ Once an organism had been characterized as a Gram positive cocci with a Gram-stained preparation, it was further identified in a series of tests, which were involved catalase, plasma coagulase, DNase, and resistance to cefoxitin $(30 \mathrm{mg}$; Oxoid, England) by using the disk diffusion method. Strains that were positive in the tests mentioned above were identified as methicillin-resistant Staphylococcus aureus (MRSA). Strains were stored frozen at $-70^{\circ} \mathrm{C}$ in Brain Heart Infusion broth (Oxoid, England) with 20\% glycerol before testing. The antibiotics used in the study were listed in the Table-I.

The minimum inhibitory concentrations (MICs) of the conventional antibiotics such as vancomycin (VA), amikacin (AK), tobramycin (TOB), azithromycin (AZM), clindamycin (CD), minocycline (MN), trimethoprimsulfamethoxazole (SXT), rifampicin (RIF), and the MICs of new generation such as telavancin (TLV), dalbavancin (DAL), daptomycin (DAP), linezolid (LNZ), ceftaroline (CPT), ceftobiprole (BPR), quinupristin-dalfopristin (QDA), and tigecycline (TGC) against 25 clinical MRSA strains were determined using E-tests in accordance with the recommendations of the manufacturing company (LIOFILCHEM $^{\circledast}$ s.r.l., Italy).

Identification of MIC values: Mueller-Hinton II Agar (MHA) (BBL ${ }^{\mathrm{TM}}$, USA) was used for determining the MICs. The strains that were incubated overnight were adjusted to the turbidity of $0.5 \mathrm{McFarland}$ in Mueller Hinton broth using densitometry (Biosan, Riga, Latvia) and $200 \mu \mathrm{L}$ of the suspension was spread onto the MHA agar plate. After drying, E-test strips were placed, and the media were incubated for $24 \mathrm{~h}$ at $35^{\circ} \mathrm{C}^{10}$ The MIC was interpreted as the value at which the inhibition zone intersected the scale on the E-strip. The determination of the susceptibilities of the strains against antimicrobials that were tested was performed according to the MIC breakpoints recommended by European Committee on Antimicrobial Susceptibility Testing (EUCAST). ${ }^{12}$ Staphylococcus aureus ATCC 29213, and Enterococcus faecalis ATCC 29212 were used for the quality control in all experiments. ${ }^{12,13}$

Antibiotic combination interactions: The combination of each antimicrobial agent with vancomycin was evaluated by the E-test. The preparation of the inoculum and the streaking of Mueller-Hinton agar plates were as previously described. The E-test strips were placed on the Mueller-Hinton agar in a cross formation with a $90^{\circ}$ angle at the intersection between the scales at their respective MICs. ${ }^{14}$ The plates were then incubated for 24 hour at $35^{\circ} \mathrm{C}$. After incubation, the zones of inhibition were read for each E-test seperately at the intersection of the zone with the E-strip as described above for the determination of the MIC results. And then, the fractional inhibitory concentration (FIC) index (FICI) values were calculated by using the following formula:

$$
\mathrm{A} / \mathrm{MIC}_{\mathrm{A}}+\mathrm{B} / \mathrm{MIC}_{\mathrm{B}}=\mathrm{FIC}_{\mathrm{A}}+\mathrm{FIC}_{\mathrm{B}}=\mathrm{FICI}
$$

In this formula;

A: identifies the MIC value of antibiotic $A$ when tested in combination with antibiotic B,

$\mathrm{MIC}_{\mathrm{A}}$ : identifies the MIC value of antibiotic $\mathrm{A}$ alone, $\mathrm{FIC}_{\mathrm{A}}$ : identifies the fractional inhibitory concentration of the antibiotic A.

B: $\quad$ identifies the MIC value of antibiotic $B$ when tested in combination with antibiotic $\mathrm{A}$,

$\mathrm{MIC}_{\mathrm{B}}$ : identifies the MIC value of antibiotic $\mathrm{B}$ alone, $\mathrm{FIC}_{\mathrm{B}}$ : identifies the fractional inhibitory concentration of the antibiotic $\mathrm{B}$.

The results of FICI $\leq 0.5$ were evaluated as synergistic, FICI $>0.5-<2$ were evaluated as 
Vancomycin combinations against MRSA

Table-I: Conventional and newer antimicrobial agents used in the study.

\begin{tabular}{ll}
\hline Conventional Antimicrobial Agents & Newer Antimicrobial Agents \\
\hline Vancomycin (VA) & Telavancin (TLV) \\
Amikacin (AK) & Dalbavancin (DAL) \\
Tobramycin (TOB) & Daptomycin (DAP) \\
Azithromycin (AZM) & Linezolid (LNZ) \\
Clindamycin (CD) & Ceftaroline (CPT) \\
Minocycline (MN) & Ceftobiprole (BPR) \\
Trimethoprim-sulfamethoxazole(SXT) & Quinupristin-Dalfopristin (QDA) \\
Rifampicin (RIF) & Tigecycline (TGC) \\
\hline
\end{tabular}

additive, FICI $\geq 2-<4$ as indifference, and FICI $\geq 4$ as antagonistic interaction. ${ }^{15}$

Ethics Approval: This being a retrospective study did not need ethics approval.

\section{RESULTS}

The $\mathrm{MIC}_{50}, \mathrm{MIC}_{90}, \mathrm{MIC}_{\text {interval }}$ values and susceptibility rates are given in the Table-II. All of the strains were found to be sensitive to vancomycin, telavancin, dalbavancin, ceftobiprole, daptomycin, linezolid, quinuprustin/dalfopristin, trimetoprim/ sulfametoxazole, rifampicin, and tigecycline. The susceptibility rate of the strains was found to be $92 \%$ for ceftaroline, $88 \%$ for minocycline,
$84 \%$ for amikacin, $76 \%$ each for clindamycin and tobramycin and $48 \%$ for azithromycin.

The interaction of vancomycin with other antimicrobials has been shown in the Table-III. Synergistic interaction was detected in five strains for VA-SXT, three strains for VA-AZM, in two each strains for VA-LNZ and VA-MN, and in one strain each for VA-DAL, VA-CD and VA-TOB combinations. The additive effect was detected in all MRSA strains (25) for VA-TLV; 24 strains for VA-DAL; 23 each for VA-CPD, VA-DAP, and VAQDA; 22 each for VA-BPR and VA-AK; 20 each for; VA-LNZ, VA-MN, and VA-TOB; 19 each for VASXT, and VA-TGC, 18 each for VA-CD and VA-

Table-II: The $\mathrm{MIC}_{50^{\prime}}, \mathrm{MIC}_{90^{\prime}}, \mathrm{MIC}_{\text {interval }}(\mathrm{mg} / \mathrm{L})$ values, sensitivity rates of $25 \mathrm{MRSA}$ strains.

\begin{tabular}{lcccc}
\hline Agents & $M C_{50}$ & $M C_{90}$ & $M I C_{\text {interval }}$ & Sensitivity $[n(\%)]$ \\
\hline VA* & 0.75 & 1 & $0.5-1$ & $25(100)$ \\
TLV** & 0.023 & 0.032 & $0.016-0.047$ & $25(100)$ \\
DAL & 0.064 & 0.064 & $0.032-0.125$ & $25(100)$ \\
BPR* & 0.75 & 1 & $0.38-1.5$ & $25(100)$ \\
CPT* & 0.5 & 1 & $0.19-1.5$ & $23(92)$ \\
DAP & 0.38 & 0.75 & $0.19-1$ & $25(100)$ \\
LNZ & 1 & 1.5 & $0.38-1.5$ & $25(100)$ \\
AZM & 24 & $>256$ & $0.5->256$ & $12(48)$ \\
CD & 0.19 & $>256$ & $0.064->256$ & $19(76)$ \\
Q/D & 1 & 1.5 & $0.5-1.5$ & $25(100)$ \\
SXT & 0.032 & 0.38 & $0.012-1$ & $25(100)$ \\
RiF & 0.012 & 0.016 & $0.006-0.094$ & $25(100)$ \\
MN & 0.094 & 3 & $0.032-16$ & $22(88)$ \\
TGC & 0.125 & 0.38 & $0.047-0.5$ & $25(100)$ \\
AK* & 2 & 32 & $1-64$ & $21(84)$ \\
TOB* & 0.5 & $>256$ & $0.125->256$ & $19(76)$ \\
\hline
\end{tabular}

*: for S. aureus; ${ }^{* *}$ : for MRSA. 
Table-III: Interactions of antibiotics with vancomycin.

\begin{tabular}{lcccc}
\hline A & Synergy & Additive & Indifference & Antagonism \\
\hline Combinations & $n(\%)$ & $n(\%)$ & $n(\%)$ & $n(\%)$ \\
\hline VA-TLV $^{1}$ & $0(0)$ & $25(100)$ & $0(0)$ & $0(0)$ \\
VA-DAL $^{2}$ & $1(4)$ & $24(96)$ & $0(0)$ & $0(0)$ \\
VA-BPR $^{3}$ & $0(0)$ & $22(88)$ & $3(12)$ & $0(0)$ \\
VA-CPT $^{4}$ & $0(0)$ & $23(92)$ & $2(8)$ & $0(0)$ \\
VA-DAP $^{5}$ & $0(0)$ & $23(92)$ & $2(8)$ & $0(0)$ \\
VA-LNZ $^{6}$ & $2(8)$ & $20(80)$ & $3(12)$ & $0(0)$ \\
VA-AZM $^{7}$ & $3(12)$ & $14(56)$ & $8(32)$ & $0(0)$ \\
VA-CD $^{8}$ & $1(4)$ & $18(72)$ & $6(24)$ & $0(0)$ \\
VA-QDA & & $23(92)$ & $2(8)$ & $0(0)$ \\
VA-SXT $^{10}$ & $0(0)$ & $19(76)$ & $1(4)$ & $0(0)$ \\
VA-RIF $^{11}$ & $5(20)$ & $18(72)$ & $7(28)$ & $0(0)$ \\
VA-MN & $0(0)$ & $20(80)$ & $3(12)$ & 00 \\
VA-TGC & $2(8)$ & $19(76)$ & $6(24)$ & $0(0)$ \\
VA-AK $^{13}$ & $0(0)$ & $22(88)$ & $2(8)$ & $1(4)$ \\
VA-TOB $^{15}$ & $0(0)$ & $20(80)$ & $4(16)$ & $0(0)$ \\
\hline
\end{tabular}

* 1:Vancomycin/telavancin, 2:Vancomycin/dalbavancin, 3: Vancomycin/ceftobiprole, 4:Vancomycin/ceftaroline, 5:Vancomycin/daptomycin, 6:Vancomycin/linezolid, 7:Vancomycin/ azithromycin, 8:Vancomycin/clindamycin, 9:Vancomycin/quinupristin\&dalfopristin, 10:Vancomycin/trimethoprim-sulfamethoxazole, 11:Vancomycin/rifampicin, 12:Vancomycin/ minocycline, 13:Vancomycin/tigecycline, 14:Vancomycin/amikacin, 15:Vancomycin/tobramycin

RIF; and 14 for VA-AZM. Indifference interaction was generally detected at low rates. Some of the results of the combination assays has been shown in the Fig.1 (a-c). No antagonistic interaction was detected in any of the combinations except against one strain for VA-AK.

\section{DISCUSSION}

Combination of synergistic antimicrobials is frequently used in order to increase the spectrum an in the treatment. A number of methods that can be used to detect in-vitro synergy between antibiotics

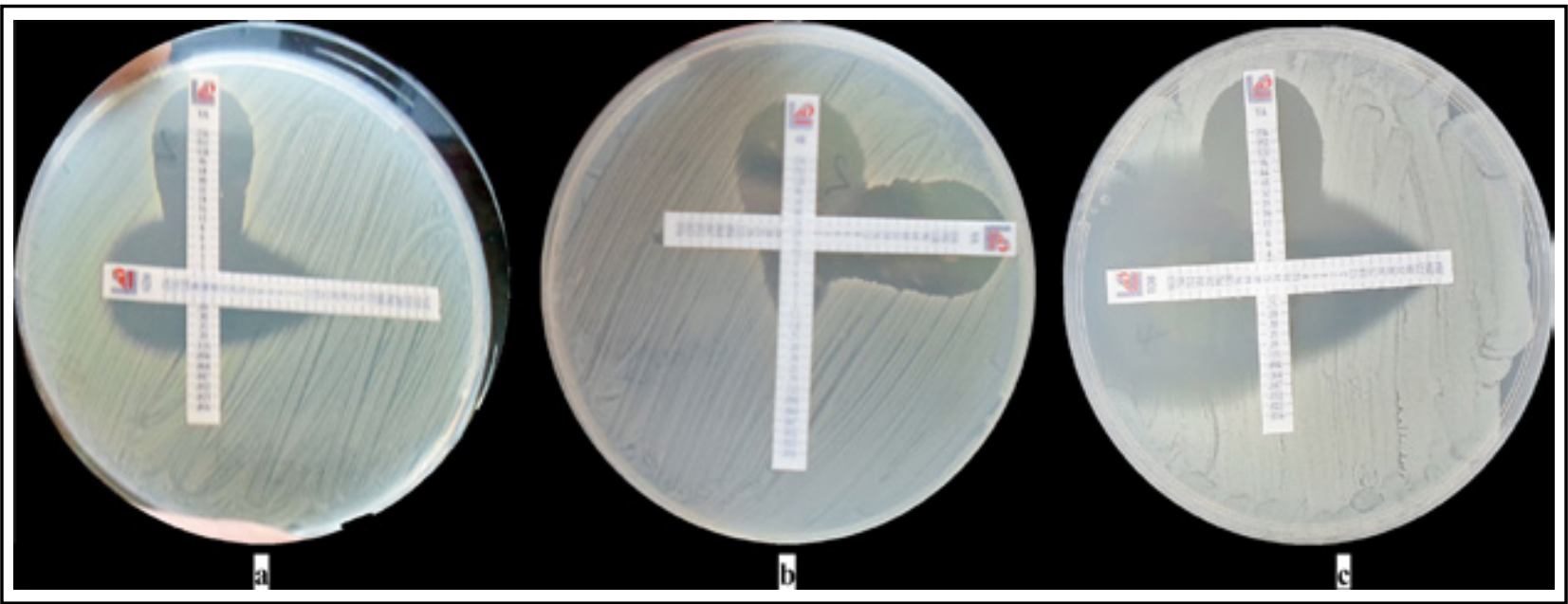

Fig.1 (a-c): Some combination results from the study. 
has been described. The E-test method has been evaluated as a good alternative for investigating the effect of the combination because of its ease of application, evaluation and good agreement with the standard checkerboard methodology. 10,14,16

In the present study in which E-test methodology was used, the synergistic results obtained from the combinations were either at low rates or none. The synergistic effect was determined as $20 \%$ for VA-STX, 12\% for VA-AZM, $8 \%$ each for VA-LNZ and for VA-MN, $4 \%$ each for VA-DAL, VA-CD and VA-TOB. Interaction results were obtained as additive at higher rates and indifference at lower rates. Parallel to the similar studies, the newer antimicrobials were found to have higher activity against MRSA strains than the convensional antimicrobials..$^{17,18}$

The results of similar studies found in literature are also summarized below. In a study that investigated the effects of vancomycin and linezolid in combination using two different methods, VA-LNZ the antibiotics were reported to demonstrate indifference effect by checkerboard method, but synergistic effect by time-kill assays. ${ }^{19}$ In other studies that was performed using timekill assay no synergy has been observed, but antagonism or indifference. ${ }^{20,21}$ In the present study, synergy, additive and indifferent interactions have been determined in 2, 20 and three strains, respectively. Similar to the results of the present study for VA-DAP combination, it has been reported that two antibiotics in combination have either indifference or additive effect, but not synergy. ${ }^{22}$ In another study, vancomycin and tigecycline combination has been tested against MRSA, and no difference in bactericidal activity was observed between combination compared with vancomycin or tigecycline alone. ${ }^{23}$ In present study, VA-TGC combination resulted in additive effect in 19 and indifference effect in six of 25 MRSA strains. Vancomycin and quinupristin/ dalfopristin has been reported to reveal both antagonistic and synergistic effects. ${ }^{24}$ In the present study, 23 additives, two indifference effects and no antagonisitic effect were observed for the combination of two antibiotics. Ceftobiprole and ceftaroline are novel, broad-spectrum cephalosporins with in vitro activities against staphylococci, including MRSA. In the present study, $\mathrm{MIC}_{50}$ and $\mathrm{MIC}_{90}$ values have been recorded as 0.75 and $1 \mathrm{mg} / \mathrm{L}$ for ceftobiprole and 0.50 and $1 \mathrm{mg} / \mathrm{L}$ for ceftaroline, respectively. Additionaly, additive effect were detected for 22 and 23 of 25 MRSA strains and indifference effect was observed for three and two strains for the vancomycin combination of ceftobiprole and ceftaroline, respectively. No synergism and antogonism was detected. The results show that both antimicrobials have excellent activities against MRSA strains. The effect of vancomycin combination with trimetoprim-sulfamethoxazole and rifampicin against MRSA strains was evaluated by da Silva LV et al. ${ }^{15}$ Synergistic effect were recorded in three strains and none, respectively. In the present study, synergistic interaction of vacomycin with trimetoprim-sulfamethoxazole and rifampicin has been detected against five MRSA strains and none, respectively. Similar to the results of our study, Miranda-Novales et al. ${ }^{25}$ reported that vancomycin and amikacin generally revealed additive effect. On the other hand, antagonistic interaction was observed for one strain in the present study.

\section{CONCLUSION}

The increase in the activity of classical and newer antibiotics studied in this study in combination with vancomycin is promising for the contributions in the treatment of infections caused by MRSA strains, taking into consideration that vancomycin has been used alone in the treatment of MRSA infections for many years, and as a result the resistance among the strains and the failure rate of the treatment has further increased over time. Additionaly, it is of importance to consider the advantage of the combination treatments to reduce and/or delay the development of resistance, it would be appropriate to carry out further studies and clarify the beneficial/supportive effects. The absence of antagonistic effect, except one strain for VA-AK combination, and generally additive / indifference results were evaluated as combinations used in the study would be useful in the treatment of MRSA infection.

Source of funding: This research was supported by the Istanbul University, Research Fund, Project number: 49403.

Conflict of interest statement: The author declares that the research was conducted in the absence of any commercial or financial relationship that could be construed as a potential conflict of interest. 


\section{REFERENCES}

1. National Nosocomial infections surveillance system. National nosocomial infections surveillance (NNIS) system report, data summary from January 1992 through June 2004. Am J Infect Control. 2004;32:470-485. doi: 10.1016/ S0196655304005425

2. Sader SH, Jones RN. Antimicrobial susceptibility of Gram-positive bacteria isolated from US medical centers: results of Daptomycin Surveillance Program (2007-2008). Diag Microbiol Infect Dis 2009;65:158-162. doi: 10.1016/j. diagmicrobio.2009.06.016

3. Shurland S, Zhan M, Bradham DD, Rogmann MC. Comparison of mortality risk associated with bacteremia due to methicillin-resistant and methicillin-susceptible Staphylococcus aureus. Infect. Control Hosp. Epidemiol. 2007;28(3):273-279. doi: 10.1086/512627

4. David MZ, Daum RS. Community-associated methicillinresistant Staphylococcus aureus: epidemiology and clinical consequences of an emerging epidemic. Clin Microbiol Rev. 2010;23(3):616-687. doi: 10.1128/CMR.00081-09

5. van HalSJ, Lodise TP, Paterson DLl. The clinical significance of vancomycin minimum inhibitory concentration in Staphylococcus aureus infections: a systemic review and meda-analysis. Clin Infect Dis. 2012; 54(6):755-771.

6. Wilcox M, Al-Obeid S, Gales A, Kozlov R, MartinezOrozco JA, Rossi $\mathrm{F}$ et al. Reporting elevated vancomycin minimum inhibitory concentration in methicillin-resistant Staphylococcus aureus: consensus by an International Working Group. Future Microbiol 2019;14(4):345-352. doi: 10.2217/fmb-2018-0346

7. Cornaglia G, Rossolini GM. Forthcoming therapeutic perspectives for infections due to multidrug-resistant Gram-positive pathogens. Clin Microbiol Infect. 2009;15(3):218-223. doi: 10.1111/j.1469-0691.2009.02740.x.

8. Lim CJ, Cheng AC, Kennon J, Spelman D, Hale D, Melican $\mathrm{G}$ et al. Prevalence of multidrug-resistant organisms and risk factors for carriage in long-term care facilities: a nested case-control study. J Antimicrob Chemother 2014;69(7):1972-1980. doi: 10.1093/jac/dku077.

9. Berti AD, Wergin JE, Girdaukas GG, Hetzel SJ, Sakoulas G, Rose WE. Altering the proclivity towards daptomycin resistance in methicillin-resistdant Staphylococcus aureus using combinations with other antibiotics. Antimicrob Agents Chemother. 2012;56(10):5046-5053. doi: 10.1128/ AAC.00502-12

10. Pillai SK, Moellering RC Jr, Eliopoulos GM. Antimicrobial combinations: In Lorian V (ed): Antibiotics in Laboratory Medicine 5. Philadelphia, Lippincott Williams and Wilkins. 2005;pp:365-440.

11. Koneman's Color Atlas and Textbook of Diagnostic Microbiology, In Washington Winn, Jr., Stephen Allen, William Janda, Elmer Koneman, Gary Procop, Paul Schreckenberger, Gail Woods (eds). Sixth edition. pp. 623671. Lippincott Williams \& Wilkins, USA, 2006.

12. EUCAST. http://www.eucast.org/clinical_ breakpoins/2016. Accessed on April 15, 2020

13. Clinical and Laboratory Standards Institute. Performance standards for antimicrobial susceptibility testing. 18th Informational supplement M100-S18. CLSI, 2008, Wayne, Pa.

14. White RL, Burgess DS, Manduru M, Bosso JA. Comparison of there different in vitro methods of detecting synergy: Time-kill, checkerboard, and E test. Antimicrob Agents Chemother 1996;40(8):1914-1918.
15. da Silva LV, Araújo MT, dos Santos KRN, Nunes APF. Evaluation of the synergistic potential of vancomycin combined with other antimicrobial agents against Methicillin-Resistant Staphylococcus Aureus and Coagulase-Negative Staphylococcus spp Strains. Mem Inst Oswaldo Cruz 2011;106(1):44-50. doi: 10.1590/s007402762011000100007.

16. Kocazeybek B, Arabaci U, Erentürk S, Akdur H. Investigation of Various Antibiotic Combinations Using the E-Test Method in Multiresistant Pseudomonas aeruginosa Strains. Chemotherapy. 2002;48(1):31-35. doi: 10.1159/000048585.

17. Isnard C, Dhalluin A, Malandain D, Bruey Q, Auzou $\mathrm{M}$, Michon J, et al. In vitro activity of novel anti-MRSA cephalosporins and comparator antimicrobial agents against staphylococci involved in prosthetic joint infections. J Glob Antimicrob Resist. 2018;13:221-225. doi: 10.1016/j.jgar.2018.01.022

18). Bal AM, David MZ, Garau J, Gottlieb T, Mazzei T, Scaglione $\mathrm{F}$, et al. Future trends in the treatment of methicillinresistant Staphylococcus aureus (MRSA) infection: An in-depth review of newer antibiotics active against an enduring pathogen. J Glob Antimicrob Resist. 2017;10:295303. doi: 10.1016/j.jgar.2017.05.019

19. Davis JS, Van Hal S, Tong SYC. Combination antibiotic treatment of serious methicillin-resistant Staphylococcus aureus infections. Semin Respir Crit Care Med. 2015;36(1):316. doi: 10.1055/s-0034-1396906

20. Grohs P, Kitzis MD, Gutmann L. In Vitro Bactericidal Activities of Linezolid in Combination With Vancomycin, Gentamicin, Ciprofloxacin, Fusidic Acid, and Rifampin Against Staphylococcus aureus. Antimicrob Agents Chemother. 2003;47(1):418-420. doi: 10.1128/aac.47.1.418420.2003

21. Singh SR, Bacon AE.3rd, Young DC, Couch KA. In vitro 24-hour time-kill studies of vancomycin and linezolid in combination versus methicillin-resistant Staphylococcus Aureus. Antimicrob Agents Chemother. 2009;53(10):44954497. doi: 10.1128/AAC.00237-09

22. Tsuji BT, Rybak MJ. Etest synergy testing of clinical isolates of Staphylococcus aureus demonstrating heterogeneous resistance to vancomycin. Diagn Microbiol Infect Dis. 2006;54(1):73-77. doi: 10.1016/j.diagmicrobio.2005.08.014

23. Rose WE, Poppens PT. Impact of biofilm on the in vitro activity of vancomycin alone and in combination with tigecycline and rifampicin against Staphylococcus aureus. J Antimicrob Chemother. 2009;63(3):485-488. doi: 10.1093/ $\mathrm{jac} / \mathrm{dkn} 513$

24. Brown J, Freeman BB III. Combining quinupristin/ dalfopristin with other agents for resistant infections. Ann Pharmacother. 2004;38(4):677-685. doi: 10.1345/aph.1D323

25. Miranda-Novales G, Leaños-Miranda BE, VilchisPerez M, Solorzano-Santos F. In vitro activity effects of combinations of cephalothin, dicloxacillin, imipenem, vancomycin and amikacin against methicillin-resistant Staphylococcus spp. strains. Ann Clin Microbiol Antimicrob. 2006;5:25. doi: 10.1186/1476-0711-5-25. 